\title{
Elovl6: a new player in fatty acid metabolism and insulin sensitivity
}

\author{
Takashi Matsuzaka • Hitoshi Shimano
}

Received: 27 August 2008 /Revised: 25 December 2008 / Accepted: 20 January 2009/Published online: 4 March 2009

(C) The Author(s) 2009. This article is published with open access at Springerlink.com

\begin{abstract}
Obesity is a major health problem in industrialized societies often associated with diabetes, insulin resistance, and hepatic steatosis. This review addresses the hypothesis that elongation of long-chain fatty acids family member 6 (Elov16) has an important role in energy metabolism and insulin sensitivity. Elovl6 is a microsomal enzyme involved in the elongation of saturated and monounsaturated fatty acids with 12, 14, and 16 carbons. Mice with targeted disruption in the gene for Elovl6 $\left(\right.$ Elovl $\left.^{\prime-}\right)$ are resistant to diet-induced insulin resistance despite their hepatosteatosis and obesity being similar to that of the wild-type mice. Protection against diet-induced insulin resistance in Elovl6 ${ }^{-1}$ mice is partially due to restoration of hepatic insulin receptor substrate-2 and suppression of hepatic protein kinase $C \varepsilon$, resulting in restoration of Akt phosphorylation. We suggest that inhibition of this elongase could be a new therapeutic approach for the treatment of insulin resistance, diabetes, cardiovascular disease, and other metabolic diseases.
\end{abstract}

Keywords Energy metabolism · Elovl6 ·

Fatty acid composition - Insulin resistance $\cdot$ Obesity

\section{Introduction}

Insulin resistance, a state of reduced responsiveness to insulin, is associated with obesity and is the major

T. Matsuzaka $\cdot H$. Shimano $(\triangle)$

Department of Internal Medicine (Endocrinology and

Metabolism), Graduate School of Comprehensive Human

Sciences, University of Tsukuba,

1-1-1 Tennodai, Tsukuba,

Ibaraki 305-8575, Japan

e-mail: hshimano@md.tsukuba.ac.jp pathogenic indicator of obesity-related diseases. Although major advances have been made in unraveling the underlying defects that cause insulin resistance, many of the pathways and regulators that connect insulin to its downstream metabolic effects are not fully understood. Epidemiological studies have demonstrated that intake of excess carbohydrate and saturated fatty acids results in accumulation of lipid, promoting a lipotoxic state, and evokes insulin resistance in the skeletal muscle, adipose tissue, and liver leading to obesity-related diseases, including the metabolic syndrome [1, 2] highlighting the importance of lipid accumulation in the pathogenesis. Previous studies on acetyl-coenzyme A (CoA) carboxylase, fatty acid synthase (FAS), and stearoyl-CoA desaturase 1 (SCD-1) indicate that endogenous fatty acid synthesis is also crucial for energy metabolism and insulin sensitivity in the liver [3-6]. Recently, we have shown that inhibition of another key lipogenic enzyme, Elovl6, which elongates long-chain saturated and unsaturated fatty acids can modulate insulin resistance in fatty livers, even with concurrent obesity [7]. In this review, we propose the hypothesis that preventing the formation of very long-chain fatty acids and manipulating the fatty acid composition by blocking this enzyme could protect against insulin resistance and obesity-related disorders, and discuss the molecular mechanism of this new paradigm.

\section{Expression and regulation of the Elovl6 gene}

Elovl6 belongs to a highly conserved family of endoplasmic reticulum enzymes involved in the formation of long-chain fatty acids [8, 9]. Functional analysis using expression experiments in cultured cells demonstrated that this enzyme specifically catalyzes the elongation of saturated and 
monounsaturated fatty acids with 12, 14, and 16 carbons, and was the last piece of the puzzle in enzymes responsible for endogenous fatty acid synthesis $[10,11]$. In mice, Elovl6 is expressed at a high level in the adrenal gland, liver, white adipose tissue, brown adipose tissue, brain, testis, and skin, where lipogenesis and steroidogenesis are active [10, 11]. Hepatic expression of the Elovl6 gene is consistently activated in the liver of transgenic mice overexpressing nuclear sterol regulatory element-binding protein (SREBP)1a, $-1 \mathrm{c}$, or $-2[10,11]$. Elovl6 mRNA levels in the liver and adipose tissue markedly elevated in a refed state after fasting [11]. Dietary polyunsaturated fatty acids cause a profound suppression of Elovl6 expression [11]. Hepatic Elovl6 expression is also highly up-regulated in leptin-deficient $o b / o b$ mice [11]. Thus, this elongase is a new member of the mammalian lipogenic enzymes regulated by SREBP-1, playing an important role in de novo synthesis of longchain saturated and monounsaturated fatty acids in conjunction with FAS and SCD-1 (Fig. 1). Promoter analysis of mouse Elovl6 identified two SREBP-binding sites: proximal SRE-1 and distal SRE-2, and Elovl6 is regulated directly and primarily by SREBP-1c [12].

Role of Elovl6 in the development of diet-induced insulin resistance Studies on Elovl6 ${ }^{-1}$ mice showed that loss of Elovl6 function increased levels of palmitate (C16:0) and palmitoleate $(\mathrm{C} 16: 1 n-7)$, but reduced levels of stearate (C18:0) and oleate (C18:1n-9), confirming that Elovl6 catalyzes the chain elongation of palmitate to stearate and the elongation of palmitoleate to vaccinate $(\mathrm{C} 18: 1 n-7)$ in vivo [7].

Fig. 1 Role of Elovl6 in mammalian fatty acid synthesis and changes of the fatty acid composition by the Elovl6 deficiency. $A C C$ acetyl-CoA carboxylase, $F A S$ fatty acid synthase, ER endoplasmic reticulum, $S C D-1$ stearoyl-CoA desaturase-1
Apart from dramatic alterations in fatty acid composition, the Elovl6 ${ }^{-1}$ mice do not differ from wild-type littermates in case of overall lipid concentration of the liver and plasma. The Elovl ${ }^{--}$mice appear grossly normal although slightly leaner than their wild-type littermates. Postprandial plasma levels of insulin and leptin are lower in the Elovl $\sigma^{--}$than that in the wild-type mice, while no significant changes are observed in plasma glucose or lipid levels.

On a high-fat, high sucrose (HF-HS) diet, the wild-type and Elovl6 ${ }^{-1}$ mice gain body weight and total body fat percentage at similar rates at identical daily food intake. The Elovl $\sigma^{--}$mice have a greater tendency to suffer from severe hepatosteatosis than the wild-type mice, as estimated by hepatic triglycerides and cholesterol. In response to diet-induced obesity, the wild-type mice exhibited a robust elevation in plasma insulin levels accompanied by a slight increase in plasma glucose, indicating the emergence of insulin resistance. However, the Elovl6 ${ }^{-1-}$ mice showed a significant reduction in plasma insulin levels compared to the wild-type mice. Insulin sensitivity, as measured by reduction in plasma glucose levels after insulin administration, was markedly reduced in the wild-type mice with an HF-HS diet, whereas the Elovl6 ${ }^{1-}$ mice showed a nearly normal response to insulin. Thus, Elovl ${ }^{-1-}$ mice are resistant to diet-induced insulin resistance, despite their hepatosteatosis and obesity being similar to that of the wild-type mice.

Elovl6 deficiency restores suppressed Akt phosphorylation in the liver, but not in the skeletal muscle and white adipose tissue. Thus, amelioration of whole-body insulin

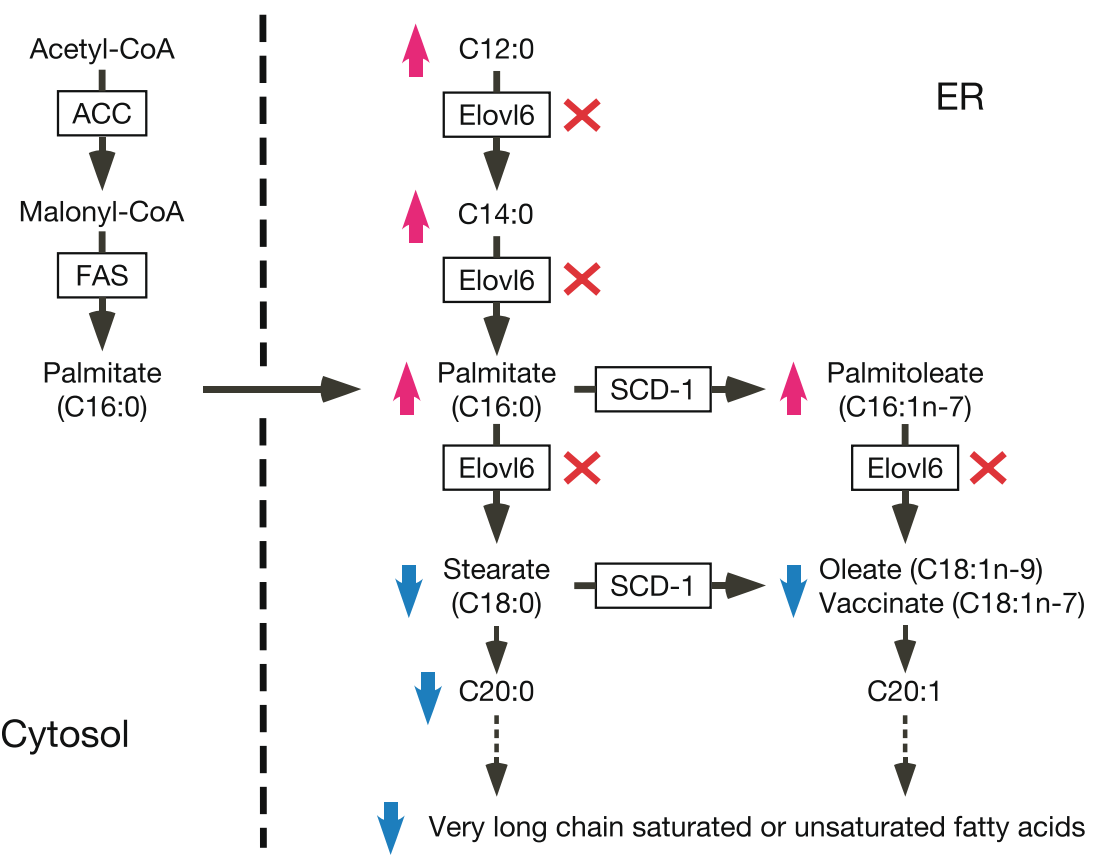


resistance in the Elovl $\sigma^{-1}$ mice can be attributed to the restoration of hepatic insulin sensitivity. Restoration of Akt phosphorylation is accompanied by increased total and phosphorylated insulin receptor substrate (IRS)-2 protein in the livers of the Elovl $\sigma^{--}$mice, whereas phosphorylated insulin receptor and IRS-1 remain suppressed by an HF-HS diet in both genotypes, demonstrating that restoration of insulin signaling is mediated by recovery of the hepatic IRS-2/Akt signaling pathway in Elovl $\sigma^{-1}$ mice (Fig. 2).

Several lines of evidence suggest that lipid metabolites, such as acyl-CoAs, diacylglycerol (DAG), and ceramides, rather than triglycerides themselves, are the determinants of the development of insulin resistance accompanying the accumulation of intracellular lipids [13-15]. DAG accumulation has been reported to be linked to increased protein kinase $\mathrm{C}$ epsilon (PKCE) activity and impaired phosphorylation of insulin receptor, IRS-1, and IRS-2 tyrosine by insulin $[13,14]$. In the wild-type mice, hepatic DAG content and $\mathrm{PKC} \varepsilon$ activity are significantly increased in the liver in response to an HF-HS diet. However, the livers of Elovl $^{1-}$ mice contain less DAG and show lower expression of PKC compared with the wild-type mice, indicating that protection against diet-induced insulin resistance in the Elovl6 ${ }^{-1}$ mice might be mediated, at least partially, through the DAG/PKCE pathway (Fig. 2).

\section{Role of Elovl6 in regulation of lipogenesis and fatty acid oxidation}

In the livers of Elovl $^{-1-}$ mice, how would energy metabolism change with changes in fatty acid composition? An HF-HS diet augmented the hepatic expression of SREBP-1c itself and its target lipogenic enzyme genes, including genes for FAS, Elov16, SCD-1, and GPAT, in the wild-type mice. The dietary induction of these lipogenic genes is suppressed in the Elovl6 ${ }^{-1}$ mice [7]. As we have previously reported, activation of SREBP-1c directly represses IRS-2, the main insulin signal mediator, and causes hepatic insulin resistance [16]. Therefore, suppression of SREBP-1c can contribute to the amelioration of hepatic insulin resistance in the Elovl6 ${ }^{-1}$ mice. Consistent with this notion, IRS-2 expression, which is completely suppressed by an HF-HS diet in the wild-type mice, is restored in the liver of Elovl6 ${ }^{-1-}$ mice (Fig. 2). Meanwhile, genes related to fatty acid oxidation that are regulated by nuclear receptor PPAR $\alpha$, such as carnitine palmitoyltransferase-1, medium-chain acyl-CoA dehydrogenase, acyl-CoA oxidase, and cytochrome P450 4a14, are induced by an HF-HS diet in the wild-type mice. However, these oxidation genes, including PPAR $\alpha$, are considerably decreased in the Elovl $\sigma^{-1}$ mice, in

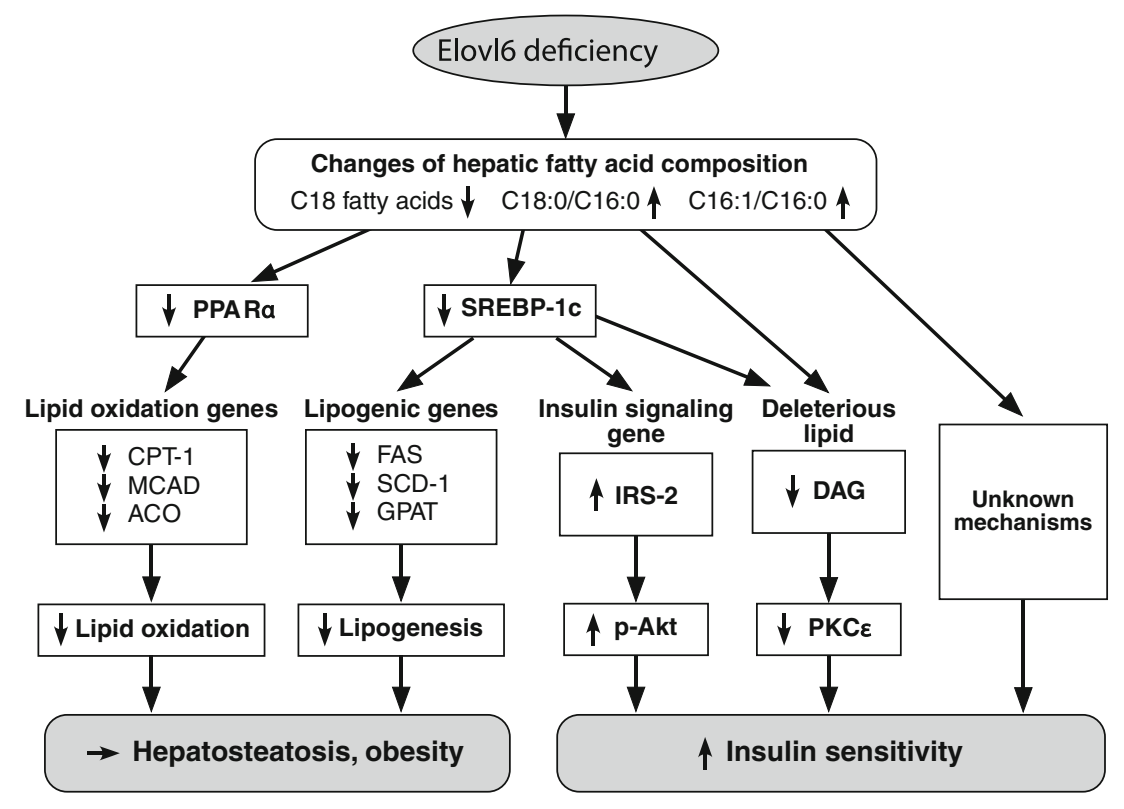

Fig. 2 Absence of Elovl6 improves hepatic insulin sensitivity without amelioration of obesity and hepatosteatosis. Elovl6 deficiency alters hepatic fatty acid composition; changes in fatty acid chain length (decrease in long-chain fatty acids more than $\mathrm{C} 18$ ) and the ratio of fatty acids (C18:0/C16:0, C16:1/C16:0) could reduce SREBP-1 and PPAR $\alpha$ in the liver. Reduction in SREBP-1 leads to decreased fatty acid synthesis via reduction of lipogenic gene expression and increases in IRS-2 level and insulin sensitivity. Reduction in lipogenesis could lead to decreased hepatic diacylglycerol content, which would lead to decreased PKCE activity and increased insulin sensitivity. Unknown mechanisms might include changes in lipid signaling and protein fatty acylation that could improve insulin signaling 
contrast to the amelioration of insulin resistance. Thus, Elovl6 deficiency leads to suppression of both synthesis and degradation of fatty acids by affecting $S R E B P-1 c$ and $P P A R \alpha$ expression (Fig. 2). The signals generated in the Elovl6 ${ }^{-1}$ mice that lead to reduced SREBP-1c and PPAR $\alpha$ expression and consequent reduction in lipid metabolism and increase in insulin sensitivity are currently being studied.

\section{Function of other Elovl enzymes}

Elovl family is composed of seven distinct fatty acid elongase subtypes (Elovl1-7) that are present in the mouse, rat, and human genomes. Each elongase has a distinct tissue distribution, and individual enzymes exhibits different fatty acid substrate preferences [8, 9, 17, 18]. Elovl1 (Ssc1) catalyze the condensation of a broad array of saturated and monounsaturated fatty acids up to C24 [19]. Elov12 (Ssc2) substrates include C20 and C22 PUFAs [19]. Elovl3 (Cig30) and Elov14 elongate different fatty acids $\leq \mathrm{C} 26$ $[20,21]$. Elovl5 is involved in the elongation of various PUFAs of C18 and C20 [22]. Elov17 has not been well characterized. In contrast to other elongases, Elovl6 expression is regulated by many dietary, hormonal, and developmental factors [11, 17, 18]. Consistent with this, Elovl6 is only elongase to connecting with de novo lipogenesis.

Deletion of the gene coding for Elovl enzymes in mice were reported in Elovl3, Elovl4, Elovl5 as well as Elovl6. Elovl3-null mice displayed a sparse hair coat, the hyperplasia of the sebaceous glands, and increased transepidermal water loss in a water barrier function test due to the decreased level of C22-24 saturated and monounsaturated fatty acids with exceptionally high levels of eicosenoic acid (C20:1) in hair lipids [23]. Elovl4 ${ }^{-1-}$ mice displayed profound skin atrophy and impaired water insulation function due to lacking ceramides with $\omega$-hydroxy very long-chain fatty acids $(\geq \mathrm{C} 28)[24,25]$. Elovl5 ${ }^{-/-}$mice demonstrated that decreased elongation of $\mathrm{C} 18: 3 n-6$ to $\mathrm{C} 20: 3 n-6$ and $\mathrm{C} 18: 4 n-3$ to $\mathrm{C} 20: 4 n-3$ and that the decreased cellular arachidonic acid $(20: 4 n-6)$ and DHA $(22: 6 n-3)$ concentrations de-represses the activation of SREBP-1c and its target genes involved in fatty acid and triglyceride synthesis, which resulted in the development of hepatic steatosis [26]. It was also reported that adenoviralmediated induction of Elovl5 activity in livers of mice increased hepatic and plasma levels of 20:3n-6 while suppressing hepatic arachidonic acid (20:4n-6) and DHA $(22: 6 n-3)$ content. Such alterations in fatty acid profile affect the expression of $\operatorname{PPAR} \alpha$-regulated genes and hepatic glucose production during fasting [27]. Elovl5 is abundant in the liver, and the gene expression is controlled by
SREBP-1 and PPAR $\alpha$. Examination of the relations between hepatic Elov15 activity and energy metabolism should provide new findings for the treatment of metabolic disease. These observations suggest that Elovl6 and possibly Elov15 are involved in central energy metabolism, whereas other Elovl enzymes are involved in regulation of longer and polyunsaturated fatty acids, which is crucial for lipid metabolism in peripheral tissues like skin. Alterations in fatty acid profile as a consequence of Elovl gene deletion, as those seen in Elovl3, Elovl4, Elovl5, and Elovl6 mutant mice, emphasize the importance of these enzymes in maintaining fatty acid homeostasis for proper cellular function and energy metabolism.

\section{New aspects of fatty acid synthesis and insulin resistance}

Palmitate has long been thought to be the major detrimental fatty acid in insulin resistance [1]. In the Elovl $\sigma^{-1}$ mice, however, the palmitate levels are increased, but insulin sensitivity is maintained, suggesting that it is the hepatic fatty acid composition, particularly the conversion of palmitate to stearate, which is crucial for insulin sensitivity than simply the accumulation of palmitate.

Recently, Cao et al. reported that the palmitoleate $(\mathrm{C} 16: 1 n-7)$ is an important signaling lipid hormone newly designated as a "lipokine," stimulating activity of muscle insulin action and influencing fat deposition in the liver [28]. Utilizing quantitative lipidomic analysis, they found that mice lacking FABP4 and FABP5, exhibiting a striking phenotype with strong protection from dietinduced obesity, insulin resistance, and fatty liver disease [29], has a fourfold higher concentration of palmitoleate in plasma, compared to wild-type mice on high-fat diet. The adipose tissue-derived palmitoleate travels to the muscle cells and liver, where it improves cell sensitivity to insulin in muscle and blocks fat accumulation in the liver. Intriguingly, our data from Elovl $\sigma^{-1}$ mice also highlights palmitoleate as a fatty acid potentially responsible for sustained insulin sensitivity. Elovl $\sigma^{--}$mice increased liver and, resultantly, plasma palmitoleate level, but skeletal muscle insulin sensitivity and hepatic triglyceride content did not altered, questioning that this particular fatty acid in circulation can simply modify peripheral tissue insulin sensitivity. The precise molecular mechanism that palmitoleate promotes the insulin sensitivity in liver and muscle is yet to be clarified. Further studies of the mice deficient in fatty acids such as FABP, Elovl6, and SCD1 are important for the clarification of this molecular mechanism.

SCD catalyzes the conversion of stearate, the end product of Elovi6, to oleate, the final product of endogenous lipogenesis. The similarity of SCD1 and Elov16 in ER 
localization, gene structures, and the coordinating functions of both proteins implicates that these genes could be derived from a common ancestral gene. Inhibition of SCD1 expression caused by Elovl6 deficiency was prominent in mice both normal and HF-HS diet [7]. Mice with global knockout of SCD1 (SCD1 $1^{-/}$mice) show decreased lipogenic gene expression and increased $\beta$-oxidation and are protected from diet-induced obesity, hepatic steatosis, and insulin resistance [5]. Liver-specific SCD1 knockout (LKO) mice are protected from high-sucrose, very low-fat diet-induced obesity and hepatic steatosis because of the decreased lipogenic gene expression without the change in the fatty acid $\beta$-oxidation gene expression [6]. It is possible that the decrease in $S C D 1$ expression could partly contribute to the insulin-sensitizing effect of Elovl6 deficiency. However, in contrast to $S C D 1^{-1-}$ and LKO mice, Elovl6 ${ }^{1-}$ mice showed sustained obesity, hepatic steatosis, and low fatty acid oxidation in liver, indicating that the mechanisms by which these two enzymes influence insulin sensitivity are not completely convergent.

Amelioration of insulin resistance in obese mice is usually accompanied by loss of fat or body weight caused by decreased food intake, enhanced lipid oxidation, and/or decreased lipogenesis $[5,30-32]$. Elovl $\sigma^{-1-}$ mice are unique in that their insulin resistance is improved without amelioration of obesity or hepatosteatosis. These data highlight the importance of tissue fatty acid composition in insulin sensitivity, especially the ratio of $\mathrm{C} 18$ to $\mathrm{C} 16$ acids, which is controlled by Elovl6 activity. The precise molecular mechanism for this is currently unknown. Distinct effects of different long fatty acids depending upon their extent of desaturation have been generally observed. However, our current studies suggest that the length of fatty acids is also important in energy metabolism and insulin sensitivity. This model aids our understanding of the mechanism by which obesity and obesityrelated disorders are sometimes dissociated, and these observations could lead to new therapeutic strategies for diabetes and cardiovascular diseases that target elongase enzymes.

For the treatment and prevention of obesity-related disorders, control of the quantity of lipid accumulating in tissue and blood is important, and thus diet has been the main target. Together with beneficial roles of some pieces of PUFA, the lessons from Elovl6 ${ }^{-1}$ mice suggest that quality aspect of lipids should be more focused for future strategy against cardiovascular diseases.

Acknowledgments We are grateful to Naoya Yahagi, Yoshimi Nakagawa, Akimitsu Takahashi, Sigeru Yatoh, Toyonori Kato, Shin Kumadaki, Ayaka Atsumi, Saori Koyasu, Junko Kato, and Konomi Mizumoto for their contribution to our work, and to Prof. Nobuhiro Yamada for continuous support.
Open Access This article is distributed under the terms of the Creative Commons Attribution Noncommercial License which permits any noncommercial use, distribution, and reproduction in any medium, provided the original author(s) and source are credited.

\section{References}

1. Riccardi G, Giacco R, Rivellese AA (2004) Dietary fat, insulin sensitivity and the metabolic syndrome. Clin Nutr 23:447-456

2. Unger RH (2003) Minireview: weapons of lean body mass destruction: the role of ectopic lipids in the metabolic syndrome. Endocrinology 144:5159-5165

3. Mao J, DeMayo FJ, Li H, Abu-Elheiga L, Gu Z, Shaikenov TE, Kordari P, Chirala SS, Heird WC, Wakil SJ (2006) Liver-specific deletion of acetyl-CoA carboxylase 1 reduces hepatic triglyceride accumulation without affecting glucose homeostasis. Proc Natl Acad Sci U S A 103:8552-8557

4. Chakravarthy MV, Pan Z, Zhu Y, Tordjman K, Schneider JG, Coleman T, Turk J, Semenkovich CF (2005) "New" hepatic fat activates PPARalpha to maintain glucose, lipid, and cholesterol homeostasis. Cell Metab 1:309-322

5. Ntambi JM, Miyazaki M, Stoehr JP, Lan H, Kendziorski CM, Yandell BS, Song Y, Cohen P, Friedman JM, Attie AD (2002) Loss of stearoyl-CoA desaturase-1 function protects mice against adiposity. Proc Natl Acad Sci U S A 99:11482-11486

6. Miyazaki M, Flowers MT, Sampath H, Chu K, Otzelberger C, Liu X, Ntambi JM (2007) Hepatic stearoyl-CoA desaturase-1 deficiency protects mice from carbohydrate-induced adiposity and hepatic steatosis. Cell Metab 6:484-496

7. Matsuzaka T, Shimano H, Yahagi N, Kato T, Atsumi A, Yamamoto $\mathrm{T}$, Inoue $\mathrm{N}$, Ishikawa $\mathrm{M}$, Okada $\mathrm{S}$, Ishigaki $\mathrm{N}$, Iwasaki H, Iwasaki Y, Karasawa T, Kumadaki S, Matsui T, Sekiya M, Ohashi K, Hasty AH, Nakagawa Y, Takahashi A, Suzuki H, Yatoh S, Sone H, Toyoshima H, Osuga J, Yamada N (2007) Crucial role of a long-chain fatty acid elongase, Elovl6, in obesity-induced insulin resistance. Nat Med 13:1193-1202

8. Leonard AE, Pereira SL, Sprecher H, Huang YS (2004) Elongation of long-chain fatty acids. Prog Lipid Res 43:36-54

9. Jakobsson A, Westerberg R, Jacobsson A (2006) Fatty acid elongases in mammals: Their regulation and roles in metabolism. Prog Lipid Res 45:237-249

10. Moon YA, Shah NA, Mohapatra S, Warrington JA, Horton JD (2001) Identification of a mammalian long chain fatty acyl elongase regulated by sterol regulatory element-binding proteins. J Biol Chem 276:45358-45366

11. Matsuzaka T, Shimano H, Yahagi N, Yoshikawa T, AmemiyaKudo M, Hasty AH, Okazaki H, Tamura Y, lizuka Y, Ohashi K, Osuga J, Takahashi A, Yato S, Sone H, Ishibashi S, Yamada N (2002) Cloning and characterization of a mammalian fatty acylCoA elongase as a lipogenic enzyme regulated by SREBPs. J Lipid Res 43:911-920

12. Kumadaki S, Matsuzaka T, Kato T, Yahagi N, Yamamoto T, Okada S, Kobayashi K, Takahashi A, Yatoh S, Suzuki H, Yamada N, Shimano H (2008) Mouse Elovl-6 promoter is an SREBP target. Biochem Biophys Res Commun 368:261-266

13. Samuel VT, Liu ZX, Qu X, Elder BD, Bilz S, Befroy D, Romanelli AJ, Shulman GI (2004) Mechanism of hepatic insulin resistance in non-alcoholic fatty liver disease. J Biol Chem 279:32345-32353

14. Samuel VT, Liu ZX, Wang A, Beddow SA, Geisler JG, Kahn M, Zhang XM, Monia BP, Bhanot S, Shulman GI (2007) Inhibition of protein kinase Cepsilon prevents hepatic insulin resistance in nonalcoholic fatty liver disease. J Clin Invest 117:739-745 
15. Holland WL, Brozinick JT, Wang LP, Hawkins ED, Sargent KM, Liu Y, Narra K, Hoehn KL, Knotts TA, Siesky A, Nelson DH, Karathanasis SK, Fontenot GK, Birnbaum MJ, Summers SA (2007) Inhibition of ceramide synthesis ameliorates glucocorticoid-, saturated-fat-, and obesity-induced insulin resistance. Cell Metab 5:167-179

16. Ide $T$, Shimano $H$, Yahagi $N$, Matsuzaka $T$, Nakakuki $M$, Yamamoto T, Nakagawa Y, Takahashi A, Suzuki H, Sone H, Toyoshima H, Fukamizu A, Yamada N (2004) SREBPs suppress IRS-2-mediated insulin signalling in the liver. Nat Cell Biol 6:351-357

17. Wang Y, Botolin D, Christian B, Busik J, Xu J, Jump D (2005) Tissue-specific, nutritional, and developmental regulation of rat fatty acid elongases. J Lipid Res. 46:706-715

18. Wang Y, Botolin D, Xu J, Christian B, Mitchell E, Jayaprakasam B, Nair MG, Peters JM, Busik JV, Olson LK, Jump DB (2006) Regulation of hepatic fatty acid elongase and desaturase expression in diabetes and obesity. J Lipid Res 47:2028-2041

19. Tvrdik P, Westerberg R, Silve S, Asadi A, Jakobsson A, Cannon B, Loison G, Jacobsson A (2000) Role of a new mammalian gene family in the biosynthesis of very long chain fatty acids and sphingolipids. J Cell Biol. 149:707-717

20. Tvrdik P, Asadi A, Kozak LP, Nedergaard J, Cannon B, Jacobsson A (1997) Cig30, a mouse member of a novel membrane protein gene family, is involved in the recruitment of brown adipose tissue. J Biol Chem. 272:31738-31746

21. Zhang K, Kniazeva M, Han M, Li W, Yu Z, Yang Z, Li Y, Metzker ML, Allikmets R, Zack DJ, Kakuk LE, Lagali PS, Wong PW, MacDonald IM, Sieving PA, Figueroa DJ, Austin CP, Gould RJ, Ayyagari R, Petrukhin K (2001) A 5-bp deletion in ELOVL4 is associated with two related forms of autosomal dominant macular dystrophy. Nat Genet 27:89-93

22. Leonard AE, Bobik EG, Dorado J, Kroeger PE, Chuang LT, Thurmond JM, Parker-Barnes JM, Das T, Huang YS, Mukerji P (2000) Cloning of a human cDNA encoding a novel enzyme involved in the elongation of long-chain polyunsaturated fatty acids. Biochem J. 350:765-770

23. Westerberg R, Tvrdik P, UndŽn A, MEEnsson J, NorlŽn L, Jakobsson A, Holleran W, Elias P, Asadi A, Flodby P, ToftgErd R,
Capecchi M, Jacobsson A (2004) Role for ELOVL3 and fatty acid chain length in development of hair and skin function. J Biol Chem 279:5621-5629

24. Cameron DJ, Tong Z, Yang Z, Kaminoh J, Kamiyah S, Chen H, Zeng J, Chen Y, Luo L, Zhang K (2007) Essential role of Elovl4 in very long chain fatty acid synthesis, skin permeability barrier function, and neonatal survival. Int J Biol Sci 3:111-119

25. Li W, Sandhoff R, Kono M, Zerfas P, Hoffmann V, Ding BC, Proia RL, Deng CX (2007) Depletion of ceramides with very long chain fatty acids causes defective skin permeability barrier function, and neonatal lethality in ELOVL4 deficient mice. Int $\mathrm{J}$ Biol Sci 3:120-128

26. Moon YA, Hammer RE, Horton JD (2009) Deletion of ELOVL5 leads to fatty liver through activation of SREBP-1c in mice. J Lipid Res 50:412-423

27. Wang Y, Torres-Gonzalez M, Tripathy S, Botolin D, Christian B, Jump DB (2008) Elevated hepatic fatty acid elongase-5 activity affects multiple pathways controlling hepatic lipid and carbohydrate composition. J Lipid Res 49:1538-1552

28. Cao H, Gerhold K, Mayers JR, Wiest MM, Watkins SM, Hotamisligil GS (2008) Identification of a lipokine, a lipid hormone linking adipose tissue to systemic metabolism. Cell 134:933-944

29. Maeda K, Cao H, Kono K, Gorgun CZ, Furuhashi M, Uysal KT, Cao Q, Atsumi G, Malone H, Krishnan B, Minokoshi Y, Kahn BB, Parker RA, Hotamisligil GS (2005) Adipocyte/macrophage fatty acid binding proteins control integrated metabolic responses in obesity and diabetes. Cell Metab 1:107-119

30. Shimada M, Tritos NA, Lowell BB, Flier JS, Maratos-Flier E (1998) Mice lacking melanin-concentrating hormone are hypophagic and lean. Nature 396:670-674

31. Loftus TM, Jaworsky DE, Frehywot GL, Townsend CA, Ronnett GV, Lane MD, Kuhajda FP (2000) Reduced food intake and body weight in mice treated with fatty acid synthase inhibitors. Science 288:2379-2381

32. Abu-Elheiga L, Matzuk MM, Abo-Hashema KA, Wakil SJ (2001) Continuous fatty acid oxidation and reduced fat storage in mice lacking acetyl-CoA carboxylase 2. Science 291:2613-2616 